\title{
Detection of Wolbachia and different trypanosome species in Glossina palpalis palpalis populations from three sleeping sickness foci of southern Cameroon
}

\author{
Sartrien Tagueu Kanté ${ }^{1}$, Trésor Melachio ${ }^{2}$, Elvis Ofon ${ }^{1}$, Flobert Njiokou ${ }^{2}$ and Gustave Simo ${ }^{1 *}$
}

\begin{abstract}
Background: African trypanosomiases are caused by trypanosomes that are cyclically transmitted by tsetse. Investigations aiming to generate knowledge on the bacterial fauna of tsetse have revealed distinct symbiotic microorganisms. Furthermore, studies addressing the tripartite association between trypanosomes-tsetse-symbionts relationship have so far been contradictory. Most studies included Sodalis glossinudius and, consequently, the association involving Wolbachia is poorly understood. Understanding the vectorial competence of tsetse requires decrypting these tripartite associations. In this study, we identified Wolbachia and trypanosomes in Glossina palpalis palpalis from three human African trypanosomiasis (HAT) foci in southern Cameroon.

Methods: Tsetse flies were captured with pyramidal traps in the Bipindi, Campo and Fontem HAT foci. After morphological identification, DNA was extracted from whole tsetse flies and Wolbachia and trypanosomes were identified by PCR using different trypanosome-specific primers and two Wolbachia-specific primers (Wolbachia surface protein and 165 rRNA genes). Statistical analyses were performed to compare the trypanosome and Wolbachia infection rates between villages and different foci and to look for an association between these microorganisms.

Results: From a total of 2122 tsetse flies, 790 G. p. palpalis were analyzed. About $25.32 \%$ of flies hosted Wolbachia and $31.84 \%$ of non-teneral flies were infected by at least one trypanosome species. There was no significant difference between the global Wolbachia prevalence revealed by the two markers while some differences were observed between HAT foci. From 248 G. p. palpalis with trypanosome infections, 62.90\% were with T. vivax, 34.68\% with T. congolense forest, $16.13 \%$ with $T$. brucei (s.l.) and $2.42 \%$ with T. congolense savannah. Of all trypanosome-infected flies, 29.84\% hosted Wolbachia and no association was observed between Wolbachia and trypanosome co-infections.

Conclusions: This study revealed differences in the prevalence of Wolbachia and trypanosomes in G. p. palpalis according to HAT foci. The use of only one marker has underestimated the prevalence of Wolbachia, thus more markers in subsequent studies may improve its detection. The presence of Wolbachia seems to have no impact on the establishment of trypanosomes in G. p. palpalis. The tripartite association between tsetse, Wolbachia and trypanosomes varies according to studied areas. Studies aiming to evaluate the genetic polymorphism of Wolbachia and its density in tsetse flies could help to better understand this association.
\end{abstract}

Keywords: Glossina palpalis palpalis, Symbiont, Wolbachia, Trypanosoma sp

\footnotetext{
* Correspondence: gsimoca@yahoo.fr; gustave.simo@univ-dschang.org

${ }^{1}$ Molecular Parasitology and Entomology Unit (MPEU), Department of

Biochemistry, Faculty of Science, University of Dschang, PO Box 67, Dschang,

Cameroon

Full list of author information is available at the end of the article
}

(c) The Author(s). 2018 Open Access This article is distributed under the terms of the Creative Commons Attribution 4.0 International License (http://creativecommons.org/licenses/by/4.0/), which permits unrestricted use, distribution, and

reproduction in any medium, provided you give appropriate credit to the original author(s) and the source, provide a link to the Creative Commons license, and indicate if changes were made. The Creative Commons Public Domain Dedication waiver (http://creativecommons.org/publicdomain/zero/1.0/) applies to the data made available in this article, unless otherwise stated. 


\section{Background}

Tsetse flies are dipteran insects of the genus Glossina. With a certain number of requirements linked to environmental factors such as the climate, the vegetation, the type of soil, the presence of domestic and/or wild fauna, and the effects of human activity, the distribution of tsetse flies is discontinuous across 37 sub-Saharan countries. Tsetse flies are the cyclical vector of African trypanosomes that cause human and animal African trypanosomiases. Two subspecies of African trypanosomes are pathogenic for humans: Trypanosoma brucei rhodesiense that causes the acute form of human African trypanosomiasis (HAT) in eastern and southern Africa, and T. b. gambiense which is responsible for the chronic form of HAT in western and central Africa [1, 2]. About 60 million people are exposed to the risk of HAT and, for the first time in 2016 and up to date, the number of reported cases is below $3000[3,4]$. In recent decades, efforts undertaken on HAT control have brought the disease under control and led to its inclusion into the WHO "roadmap for elimination of neglected tropical diseases" with a target set to eliminate HAT as a public health problem by 2020 [1].

Alongside T. $b$. rhodesiense and T. $b$. gambiense, other African trypanosomes including $T . b$. brucei, T. congolense, $T$. vivax and $T$. simiae are responsible for the animal African trypanosomiasis (AAT) or "nagana" in animals. AAT is one of the biggest constraints to livestock production and a threat to food security in sub-Saharan Africa. Human and animal trypanosomiases have impacts on human and animal health, but also on animal productivity and, therefore, the peasant economy. In both human and animal trypanosomiases, tsetse flies play a key role in the transmission of parasites between different vertebrate hosts. To achieve HAT elimination and boost AAT control, the integration of vector control as component of new control strategies is becoming crucial. A better understanding of how trypanosomes develop in tsetse flies appears to be an important step in the process leading to the development of innovative vector control strategies. In recent decades, growing interests have been focused on tripartite interactions between trypanosomes, tsetse fly and tsetse-associated symbiotic microorganisms. Currently, three symbiotic microorganisms including Wigglesworthia glossinidia, Sodalis glossinidius and Wolbachia have been reported to be associated with tsetse flies. While W. glossinidia is an obligate primary symbiont, S. glossinidius is a secondary and a non-essential symbiont which seems to affect vector competence of tsetse by favoring the midgut establishment of trypanosomes through a complex biochemical mechanism. Wolbachia spp. are also non-essential symbionts that infect a wide range of invertebrates. Abundant in both male and female germ-cells and also in the somatic tissues, Wolbachia spp. are found in a wide range of arthropods [5] and nematodes [6]. Transmitted vertically from mother to offspring [7], Wolbachia can protect their hosts against viral pathogens [8]. It has the ability to induce cytoplasmic incompatibility that leads to embryonic death in tsetse flies $[9,10]$. Investigations on Wolbachia in tsetse populations may improve vector control through the development of transgenic tsetse with the ability to release specific molecules that can interfere with the establishment of trypanosomes. Previous studies reported Wolbachia in several tsetse species from insectariums and few wild tsetse populations such as Glossina morsitans morsitans, G. m. centralis, G. f. fuscipes, G. austeni, G. pallidipes and G. brevipalpis [11-15]. Investigations on the tripartite association between trypanosomes, tsetse fly and its symbiotic microorganisms reported contrasting results. For instance, Alam et al. [14] reported a negative association between Wolbachia and trypanosome infections in G. f. fuscipes, suggesting that the presence of Wolbachia could prevent trypanosome infections. Despite these interesting results, little investigation has been undertaken on the tripartite association between tsetse fly, trypanosomes and Wolbachia, and therefore this tripartite association is not well understood. A better understanding of this association requires the collection of more data on trypanosome and symbiont infections in different tsetse species from various tsetse infested areas. In tsetse flies of the palpalis group, investigations on the tripartite association were focused essentially on $S$. glossinidius and trypanosomes [16, 17]. These investigations revealed a positive association between the presence of S. glossinidius and trypanosome infections $[16,17]$. However, there is currently very little, if any, data on the tripartite association involving Wolbachia in tsetse of the palpalis group.

In the present study, Wolbachia and different trypanosome species were identified in wild populations of G. p. palpalis caught in three sleeping sickness foci of southern Cameroon with the final goal of generating data that may shed more light on the tripartite association and help to understand the impact of Wolbachia infections on the transmission of African trypanosomes.

\section{Methods}

\section{Study zones}

This study was performed in the Bipindi, Campo and Fontem HAT foci located in the forest region of southern Cameroon (Fig. 1). The Bipindi and Campo HAT foci are located in the Ocean Division of the South Region of Cameroon. The Campo HAT focus offers several types of biotopes (farmland, swampy areas and equatorial forest) while the Bipindi HAT focus shows a typical forest bioecological environment.

The Bipindi $\left(3^{\circ} 2^{\prime} 00^{\prime \prime} \mathrm{N}, 10^{\circ} 22^{\prime} 00^{\prime \prime} \mathrm{E}\right)$ HAT focus has been known since 1920 [18]. It covers several villages located 


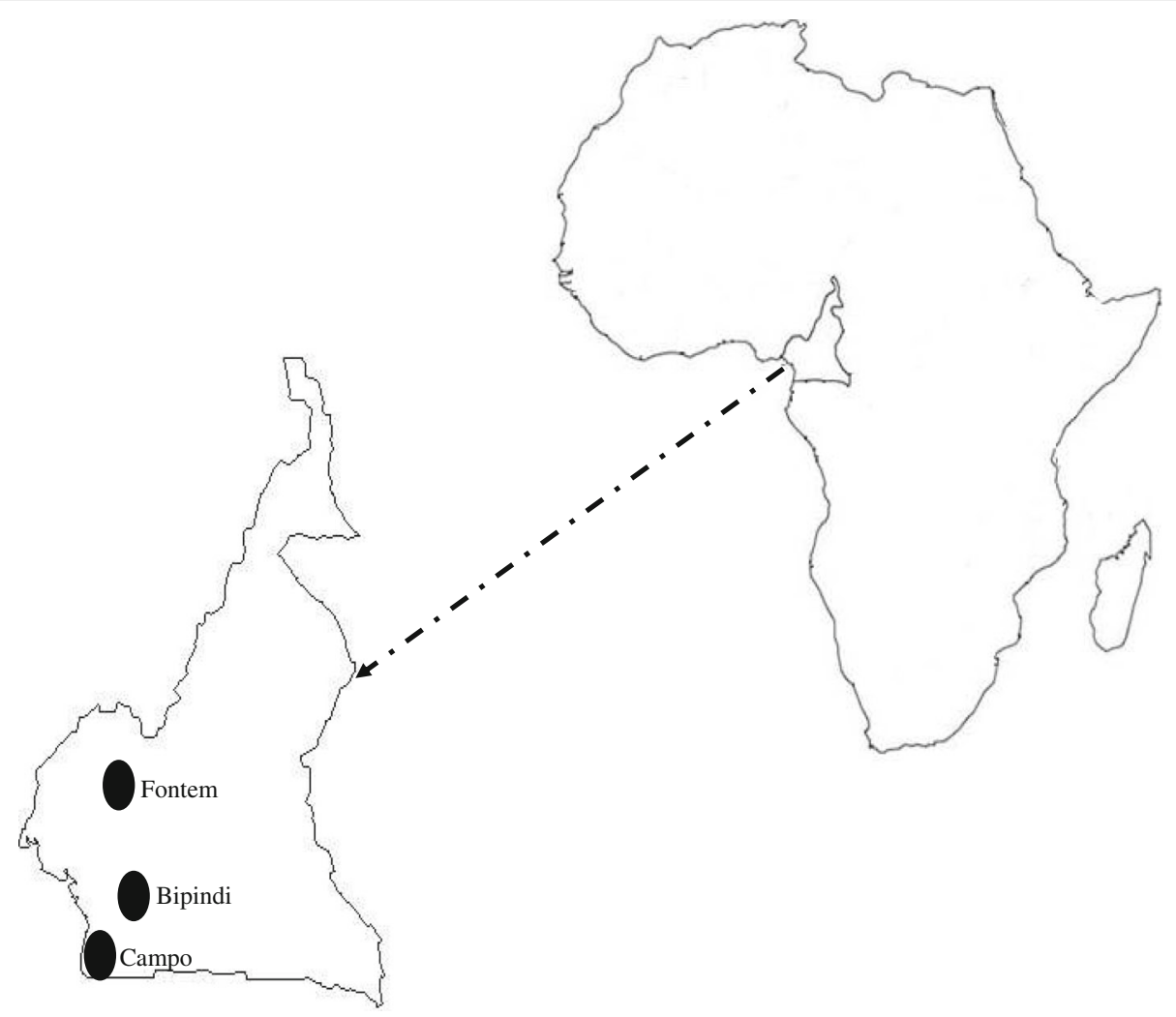

Fig. 1 Map showing sleeping sickness foci where tsetse flies were caught (circles)

along the roads and its bio-ecological environment is characterized typically by an equatorial forest with farmland along the roads and the villages. The wild fauna composition is highly diversified [19]. Peasant agriculture, hunting, fishing and breeding of livestock are the main socioeconomic activities. The focus is surrounded by hills and has a dense hydrographic network with many rivers crossing farmlands. The bioclimatic environment offers suitable habitats for tsetse flies.

The Campo HAT focus $\left(2^{\circ} 22^{\prime} 00^{\prime \prime} \mathrm{N}, 9^{\circ} 49^{\prime} 00^{\prime \prime} \mathrm{E}\right)$ lies along the Atlantic coast and extends along the Ntem River which constitutes the border of Cameroon and Equatorial Guinea. This focus is characterized by an equatorial rainforest with a hydrographic network containing several rivers and swampy areas. Its fauna composition is highly diversified. The climate is typical maritime equatorial comprising four seasons. The main activities of the inhabitants are fishing, picking fruits, hunting and farming.

The Fontem HAT focus $\left(5^{\circ} 40^{\prime} 12^{\prime \prime} \mathrm{N}, 9^{\circ} 55^{\prime} 33^{\prime \prime} \mathrm{E}\right)$ is located in the Lebialem division of the southwest region of Cameroon. It is characterized by a tropical humid climate, having an irregular relief with many hills and valleys that are crossed by fast-flowing streams. The main population activities are subsistence agriculture, palm oil extraction, animal husbandry and small-scale poultry farming. The dense population of humans, domestic animals (dogs, pigs, sheep and goats) and tsetse flies are found scattered in the pre-forest/forest vegetation of the valleys and hills.

\section{Trapping of tsetse flies}

Tsetse flies were collected during four entomological surveys in the three HAT foci of Cameroon. During the first survey in the Campo HAT focus in 2012, tsetse flies were trapped at Akak, Campo beach, Ipono and Mabiogo villages. During the second survey in 2015, tsetse flies were trapped at Bidjouka, Ebiminbang and Lambi villages of the Bipindi HAT focus. The third and fourth surveys were performed in 2015 and 2017 at Bechati, Besali Folepi and Menji villages in the Fontem HAT focus. During each survey, pyramidal traps [20] were set for 4 consecutive days. In total, 197 traps were set up: 105 at Campo, 50 at Bipindi and 42 at Fontem. The geographical coordinates of each trap were recorded using a global positioning system (GPS). Tsetse flies were collected twice a day. All collected flies were morphologically identified, counted and sorted into teneral and non-teneral flies as described by Pollock [21]. Thereafter, each identified fly was put into a microtube containing $95 \%$ ethanol. In the field, microtubes were kept at room temperature, and in the laboratory they were stored at $-20^{\circ} \mathrm{C}$. 


\section{DNA extraction}

DNA was extracted from whole tsetse fly using the cetyl trimethyl ammonium bromide (CTAB) method as described by Navajas et al. [22]. Briefly, the alcohol preserving each fly was evaporated by incubating the opened microtubes at $80{ }^{\circ} \mathrm{C}$ in an oven for about $1 \mathrm{~h}$. Thereafter, each tsetse fly was disrupted with a pestle in CTAB buffer (CTAB 2\%; $1 \mathrm{M}$ Tris, pH 8; 0.5 M EDTA $\mathrm{pH} 8 ; 5 \mathrm{M} \mathrm{NaCl})$. The disrupted fly was incubated at 60 ${ }^{\circ} \mathrm{C}$ for $30 \mathrm{~min}$ before the addition of chloroform/isoamylic alcohol mixture $(24 / 1, \mathrm{~V} / \mathrm{V})$. DNA was precipitated by addition of isopropanol $(\mathrm{V} / \mathrm{V})$ followed by centrifugation at $13,000 \times \mathrm{rpm}$ for $15 \mathrm{~min}$. The resulting DNA pellets were washed twice with cold $70 \%$ ethanol and then dried overnight at room temperature. DNA pellets were finally re-suspended in $50 \mu \mathrm{l}$ of sterile water before storing at $-20{ }^{\circ} \mathrm{C}$ until use.

\section{Molecular identification of Wolbachia}

The identification of Wolbachia was performed using two sets of primers. The first set of primers, wspec F1 (5'-YAT ACC TAT TCG AAG GGA TAG-3') and wspec R1 (5'-AGC TTC GAG TGA AAC CAA TTC-3'), described by Werren \& Windsor [23], amplifies a fragment of the $16 S$ rRNA gene. The second set of primers, wsp $\mathrm{F}_{1}$ (5'-GTC CAA TAR STG ATG ARG AAA C-3') and wsp $\mathrm{R}_{1}$ (5'-CYG CAC CAA YAG YRC TRT AAA-3'), described by Baldo et al. [24], amplifies a fragment of the Wolbachia surface protein gene. All PCR reactions were performed in a final volume of $15 \mu \mathrm{l}$ containing 3 $\mu \mathrm{l}$ of DNA extract, $1.5 \mu \mathrm{l}$ of $10 \times$ PCR reaction buffer, 2 $\mathrm{mM} \mathrm{MgCl}_{2}, 20$ pmol of each primer, $200 \mathrm{mM}$ of each dNTP and 0.3 units of Taq DNA polymerase (New England Biolabs, Massachusetts, USA; 5U/ $\mu$ l). The amplification program comprised an initial denaturation step at $94{ }^{\circ} \mathrm{C}$ for $3 \mathrm{~min}$ followed by 37 amplification cycles of denaturation at $94{ }^{\circ} \mathrm{C}$ for $30 \mathrm{~s}$, annealing at $54{ }^{\circ} \mathrm{C}$ (wspec) or $53{ }^{\circ} \mathrm{C}$ (wsp) for $30 \mathrm{~s}$, and extension at $72{ }^{\circ} \mathrm{C}$ for $1 \mathrm{~min}$. A final extension was performed at $72{ }^{\circ} \mathrm{C}$ for $5 \mathrm{~min}$.

At the end of PCR reactions, $10 \mu \mathrm{l}$ of amplified product was analyzed by electrophoresis on $2 \%$ agarose gel containing ethidium bromide. Each gel was visualized under UV light and then photographed.

\section{Detection of trypanosomes}

Different trypanosome species including T. brucei (s.l.), $T$. vivax, $T$. congolense forest type and $T$. congolense savannah type were investigated. Trypanosome identification was performed as previously described by Herder et al. [25] using the primers TCF1/2 (5'-GGA CAC GCC AGA AGG TAC TT-3'; 5'-GTT CTC GCA CCA AAT CCA AC-3') for $T$. congolense forest type [26], TCN1/2 (5'-TCG AGC GAG AAC GGG CAC TTT GCG A-3'; 5'-ATT AGG GAC AAA CAA ATC CCG CAC A-3') for
T. congolense savannah type [27], TBR1/2 (5'-CGA ATG AAT ATT AAA CAA TGC GCA G-3'; 5'-AGA ACC ATT TAT TAG CTT TGT TGC-3') for T. brucei (s.l.) [26] and TVW1/2 (5'-CTG AGT GCT CCA TGT CCC AC-3'; 5'-CCA CCA GAA CAC CAA CCT GA-3') for $T$. vivax [26]. The amplification reaction was carried out in a final volume of $15 \mu \mathrm{l}$ containing $1.5 \mu \mathrm{l}$ of $10 \times$ PCR reaction buffer, $1.5 \mathrm{mM} \mathrm{MgCl}, 0.5 \mu \mathrm{l}$ of dNTPs $(200 \mathrm{mM}$ for each dNTP), $1 \mu \mathrm{l}$ (10 pmol) of each primer, $0.3 \mathrm{U}$ of Taq DNA polymerase (New England Biolabs; $5 \mathrm{U} / \mu \mathrm{l}), 3$ $\mu \mathrm{l}$ of DNA extract, and nuclease-free water. The amplification program comprised an initial denaturation step at $94{ }^{\circ} \mathrm{C}$ for $5 \mathrm{~min}$, followed by 40 amplification cycles of denaturation at $94{ }^{\circ} \mathrm{C}$ for $30 \mathrm{~s}$, annealing at $60{ }^{\circ} \mathrm{C}$ for 30 $\mathrm{s}$ for the four trypanosome species investigated in this study, and an extension step at $72{ }^{\circ} \mathrm{C}$ for $1 \mathrm{~min}$. A final extension was performed at $72{ }^{\circ} \mathrm{C}$ for $10 \mathrm{~min}$.

Amplified products were resolved on $2 \%$ agarose gel containing ethidium bromide and visualized under UV light.

\section{Statistical analysis}

Statistical analyses were performed using the $\mathrm{R}$ 3.4.1 [28]. A Chi-square test was used to compare, between foci, the infection rates of Wolbachia sp. and different trypanosome species. The differences were considered significant when $P$-values were lower than 0.05 . To analyze the relationship between Wolbachia sp. and trypanosome infections, a generalized linear model (package stats in R) was used with 95\% confidence intervals (CIs). Trypanosoma vivax was excluded for these analyses because its life-cycle is restricted to the mouthparts of tsetse flies.

\section{Results}

\section{Entomological surveys}

From the 197 traps used in this study, a total of 2122 tsetse flies were collected during the four entomological surveys: 1216 (57.3\%) tsetse flies were caught in the Bipindi HAT focus, 632 (29.78\%) in the Campo focus and $274(12.91 \%)$ in the Fontem focus. Four different tsetse species and subspecies including G. caliginea, G. tabaniformis, G. p. palpalis and G. p. pallicera were identified. Glossina p. palpalis was the only tsetse subspecies caught in the Fontem HAT focus. In the Campo HAT focus, 632 tsetse flies were identified, of which 619 (97.94\%) were G. p. palpalis, 9 (1.42\%) G. pallicera, 3 $(0.47 \%)$ G. tabaniformis and $1(0.16 \%)$ G. caliginea. In the Bipindi HAT focus, 1216 tsetse flies were identified, of which 1208 (99.34\%) were G. p p palpalis and 8 $(0.66 \%)$ G. pallicera. In the three HAT foci, $34(1.6 \%)$ teneral flies were identified: $1(0.05 \%)$ at Bipindi, 24 $(1.13 \%)$ at Campo and $9(0.42 \%)$ at Fontem. For the molecular identification of Wolbachia and different 
trypanosome species, 790 (37.23\%) G. p. palpalis were randomly selected.

\section{Molecular identification of Wolbachia}

From 790 tsetse flies that were randomly selected and analyzed by two set of primers (wspec $F_{1} /$ wspec $R_{1}$ and wsp $\mathrm{F}_{1} /$ wsp $\mathrm{R}_{1}$ ), at least one of the two markers identified Wolbachia infections in a total of 200 tsetse flies. This gave a global infection rate of $25.32 \%$ (200/790) (Table 1).

The highest infection rate of $71.83 \%$ was observed in tsetse flies caught at Menji in the Fontem HAT focus and the lowest infection rate of $13.64 \%$ in flies caught at Mabiogo in the Campo HAT focus. Between HAT foci, a significant difference $\left(\chi^{2}=6.9543, d f=2\right.$, $P=0.0309)$ was observed in the Wolbachia infection rates. Similar results were observed between villages of the same HAT focus, except in the Bipindi focus where the difference in the Wolbachia infection rates was not significant $\left(\chi^{2}=1.1123, d f=2, P=0.5734\right)$ (Table 1).

\section{Comparison of results generated by 165 rDNA and WSP markers}

Of the 200 tsetse flies with Wolbachia infections, 130 (65\%) were positive for the 165 DNA marker and 121 (60.5\%) for WSP. Fifty-one (25.5\%; 51/200) of these infections were simultaneously identified by both $16 S$ and
WSP markers (Table 2). However, no significant difference $\left(\chi^{2}=0.3837, d f=1, P=0.5357\right)$ was observed between the number of Wolbachia infections identified by these two markers.

In the Bipindi and Campo HAT foci, WSP appeared more sensitive because 40 (19.14\%) and 62 (20.19\%) Wolbachia infections, respectively, were identified by this marker while only $26(12.44 \%)$ and 29 (9.45\%) infections, respectively, were identified by $16 S$ in the same focus. Wolbachia infections simultaneously identified by the two markers in the Bipindi and Campo HAT foci were $12(5.74 \% ; 12 / 209)$ and $29(9.45 \% ; 29 / 307)$, respectively. The difference in sensitivity between these markers was significant in the Campo $\left(\chi^{2}=14.049, d f=1\right.$, $P=0.0002)$ HAT focus, but not in the Bipindi $\left(\chi^{2}=3.5265\right.$, $d f=1, P=0.0604$ ) HAT focus (Table 2).

In the Fontem HAT focus, $16 \mathrm{~S}$ rDNA detected significantly $\left(\chi^{2}=40.269, d f=1, P<0.0001\right)$ more infections than WSP (Table 2): 75 (27.37\%) Wolbachia infections were identified by $16 S$ compared to 19 (6.93\%) identified by WSP. In this focus, only 3.65\% (10/274) of Wolbachia infections were simultaneously detected by $16 S$ and WSP (Table 2).

Regardless of the marker used in this study (16S or WSP), a significant difference $\left(\chi^{2}=22.831, d f=2, P<0.0001\right.$ for WSP; $\chi^{2}=8.296, d f=2, P=0.0158$ for $16 S \mathrm{rDNA}$ ) was observed in the Wolbachia infection rates between all the three HAT foci (Table 2).

Table 1 Infection rates of Wolbachia according to villages and different HAT foci

\begin{tabular}{|c|c|c|c|c|c|}
\hline Focus & Village & No. of flies captured & No. of flies analyzed & No. of flies hosting Wolbachia (\%) & $95 \% \mathrm{Cl}$ \\
\hline \multirow[t]{5}{*}{ Bipindi } & Bidjouka & 600 & 77 & $17(22.08)$ & $14.27-32.54$ \\
\hline & Ebimimbang & 122 & 46 & $14(30.43)$ & $19.08-44.81$ \\
\hline & Lambi & 486 & 86 & $23(26.74)$ & $18.53-36.95$ \\
\hline & Total (1) & 1208 & 209 & $54(25.84)$ & \\
\hline & $P$-value & & & 0.5734 & \\
\hline \multirow[t]{6}{*}{ Campo } & Akak & 212 & 142 & $29(20.42)$ & $14.61-27.79$ \\
\hline & Campo beach & 157 & 27 & $12(44.44)$ & $27.59-62.69$ \\
\hline & Ipono & 72 & 72 & $12(16.67)$ & $9.8-26.91$ \\
\hline & Mabiogo & 178 & 66 & $9(13.64)$ & 7.34-23.93 \\
\hline & Total (2) & 619 & 307 & $62(20.2)$ & \\
\hline & $P$-value & & & 0.0068 & \\
\hline \multirow[t]{6}{*}{ Fontem } & Bechati & 54 & 54 & $9(16.67)$ & $9.02-28.74$ \\
\hline & Besali & 4 & 4 & $1(25)$ & \\
\hline & Folepi & 145 & 145 & $23(15.86)$ & $10.81-22.68$ \\
\hline & Menji & 71 & 71 & $51(71.83)$ & $60.46-80.96$ \\
\hline & Total (3) & 274 & 274 & $84(30.66)$ & \\
\hline & $P$-value & & & $<2.2 \mathrm{e}-16$ & \\
\hline \multicolumn{2}{|c|}{ Total $(1)+(2)+(3)$} & 2101 & 790 & $200(25.32)$ & \\
\hline \multicolumn{2}{|l|}{$P$-value } & & & 0.0309 & \\
\hline
\end{tabular}


Table 2 Wolbachia infections according to different HAT foci

\begin{tabular}{|c|c|c|c|c|c|c|c|c|}
\hline \multirow[t]{2}{*}{ HAT foci } & \multirow{2}{*}{$\begin{array}{l}\text { No. of samples } \\
\text { analysed }\end{array}$} & \multicolumn{7}{|c|}{ Wolbachia infections } \\
\hline & & 165 rDNA (\%) & $95 \% \mathrm{Cl}$ & WSP (\%) & $95 \% \mathrm{Cl}$ & $P$-value & $\begin{array}{l}165 \text { rDNA } \\
\text { and WSP (\%) }\end{array}$ & $95 \% \mathrm{Cl}$ \\
\hline Bipindi & 209 & $26(12.4)$ & $8.6-17.6$ & $40(19.1)$ & $14.4-25.0$ & 0.0604 & $12(5.7)$ & $3.3-9.8$ \\
\hline Campo & 307 & $29(9.5)$ & $6.7-13.2$ & $62(20.2)$ & $16.1-25.0$ & 0.0002 & $29(9.5)$ & $6.7-13.2$ \\
\hline Fontem & 274 & $75(27.4)$ & $22.4-32.9$ & $19(6.9)$ & $4.5-10.6$ & $2.213 e-10$ & $10(3.7)$ & $209-6.6$ \\
\hline Total & 790 & $130(16.5)$ & & $121(15.3)$ & & 0.5357 & $51(6.5)$ & \\
\hline$P$-value & & 8.466e-09 & & 0.00001 & & & 0.0158 & \\
\hline
\end{tabular}

Abbreviations: \%, Wolbachia infection rate, $\mathrm{Cl}$ confidence interval

\section{Molecular detection of different trypanosome species}

From 790 tsetse flies randomly selected, 11 were teneral flies. These 11 flies were excluded from the identification of trypanosomes because they had never taken a blood meal. From the remaining 779 non-teneral flies that were subjected to molecular identification of trypanosomes, 248 (31.84\%) were infected with at least one trypanosome species: 156 (62.90\%) with T. vivax; 86 (34.68\%) with $T$. congolense forest type; 40 (16.13\%) T. brucei (s.l.) and $6(2.42 \%)$ T. congolense savannah type (Table 3). Between villages of the same HAT focus, no significant difference was observed in the infection rates of different trypanosome species except for $T$. congolense forest type $\left(\chi^{2}=10.254, d f=2, P=0.0059\right)$ in the Fontem HAT focus (Table 3 ).

Trypanosoma vivax was found in tsetse flies caught in the three HAT foci. Its prevalence in tsetse flies was $21.64 \%$ in the Campo HAT focus, $20.1 \%$ in the Bipindi HAT focus and $18.11 \%$ in the Fontem HAT focus. The highest infection rate of $27.27 \%$ was observed at

Table 3 Trypanosome infections according to villages and different HAT foci

\begin{tabular}{|c|c|c|c|c|c|c|c|c|}
\hline \multirow[t]{2}{*}{ Focus } & \multirow[t]{2}{*}{ Village } & \multirow{2}{*}{$\begin{array}{l}\text { No. of flies } \\
\text { captured }\end{array}$} & \multirow{2}{*}{$\begin{array}{l}\text { No. of flies } \\
\text { analyzed }\end{array}$} & \multicolumn{5}{|c|}{ Trypanosome infections [95\% Cl] } \\
\hline & & & & $T+(\%)$ & $\mathrm{Tb}($ s.l.) (\%) & Tcf (\%) & Tcn (\%) & Tv (\%) \\
\hline \multirow[t]{5}{*}{ Bipindi } & Bidjouka & 600 & 77 & $17(22.1)$ & $0(0)$ & $0(0)$ & $0(0)$ & $17(22.1)$ \\
\hline & Ebimimbang & 122 & 46 & $9(19.6)$ & $0(0)$ & $0(0)$ & $0(0)$ & $9(19.6)$ \\
\hline & Lambi & 486 & 86 & $16(18.6)$ & $0(0)$ & $0(0)$ & $0(0)$ & $16(18.6)$ \\
\hline & Total (1) & 1208 & 209 & $\begin{array}{l}42(20.1) \\
{[15.2-26.1]}\end{array}$ & $0(0)$ & $0(0)$ & $0(0)$ & $\begin{array}{l}42(20.1) \\
{[15.2-26.1]}\end{array}$ \\
\hline & $P$-value & & & 0.854 & & & & 0.854 \\
\hline \multirow[t]{6}{*}{ Campo } & Akak & 212 & 141 & $42(29.8)^{a}$ & $14(9.9)$ & $11(7.8)$ & $2(1.4)$ & $28(19.9)$ \\
\hline & Campo beach/center & 157 & 27 & $9(33.3)^{a}$ & $2(7.4)$ & $1(3.7)$ & $0(0)$ & $7(25.9)$ \\
\hline & Ipono & 72 & 71 & $18(25.4)^{\mathrm{a}}$ & $0(0)$ & $4(5.6)$ & $4(5.6)$ & $13(18.3)$ \\
\hline & Mabiogo & 178 & 66 & $37(56.1)^{\mathrm{a}}$ & $24(36.4)$ & $1(1.5)$ & $0(0)$ & $18(27.3)$ \\
\hline & Total (2) & 619 & 305 & $\begin{array}{l}106(34.8) \\
{[29.6-40.3]^{\mathrm{a}}}\end{array}$ & $\begin{array}{l}40(13.1) \\
{[9.8-17.4]}\end{array}$ & $\begin{array}{l}17(5.6) \\
{[3.51-8.74]}\end{array}$ & $\begin{array}{l}6(2.0) \\
{[0.9-4.2]}\end{array}$ & $\begin{array}{l}66(21.6) \\
{[17.4-26.6]}\end{array}$ \\
\hline & $P$-value & & & 0.0005 & & & & 0.521 \\
\hline \multirow[t]{6}{*}{ Fontem } & Bechati & 54 & 53 & $18(34.0)^{\mathrm{a}}$ & $0(0)$ & 9 (16.98) & $0(0)$ & $11(20.8)$ \\
\hline & Besali & 4 & 4 & $1(25)^{a}$ & $0(0)$ & $1(25)$ & $0(0)$ & $1(25)$ \\
\hline & Folepi & 145 & 138 & $48(34.9)^{\mathrm{a}}$ & $0(0)$ & $31(22.46)$ & $0(0)$ & $22(15.9)$ \\
\hline & Menji & 71 & 70 & $33(47.1)^{\mathrm{a}}$ & $0(0)$ & $28(40)$ & $0(0)$ & $14(20)$ \\
\hline & Total (3) & 274 & 265 & $\begin{array}{l}100(37.7) \\
{[32.1-43.7]^{a}}\end{array}$ & $0(0)$ & 69 (26.04) [21.1-31.6] & $0(0)$ & $\begin{array}{l}48(18.1) \\
{[13.9-23.2]}\end{array}$ \\
\hline & $P$-value & & & 0.1774 & & 0.0059 & & 0.6512 \\
\hline \multicolumn{2}{|c|}{ Total $(1)+(2)+(3)$} & 2101 & 779 & $\begin{array}{l}248(31.8) \\
{[28.7-35.2]^{a}}\end{array}$ & $\begin{array}{l}40(5.1) \\
{[3.8-6.9]}\end{array}$ & 86 (11.0) [9.0-13.4] & $\begin{array}{l}6(0.8) \\
{[0.35-1.7]}\end{array}$ & $\begin{array}{l}156(20.1) \\
{[17.4-23.0]}\end{array}$ \\
\hline \multicolumn{2}{|l|}{$P$-value } & & & $7.76 \mathrm{e}-05$ & & & & 0.5596 \\
\hline
\end{tabular}

${ }^{\mathrm{a}}$ Parts of tsetse flies with co-infections of different trypanosome species

Abbreviations: \% trypanosome infection rate, T+ tsetse flies with at least one trypanosome infection, Tb (s.l.) Trypanosoma brucei sensu lato, Tcf Trypanosoma congolense forest type, Tcn Trypanosoma congolense savannah type, TV Trypanosoma vivax, Cl confidence interval 
Mabiogo in the Campo HAT focus and the lowest infection rate of $15.94 \%$ in tsetse captured at Folepi in the Fontem HAT focus (Table 3). Between HAT foci, no significant difference $\left(\chi^{2}=1.161, d f=2, P=0.5596\right)$ was observed in the infection rates of $T$. vivax.

Trypanosoma congolense savannah type and T. brucei (s.l.) were found in tsetse from the Campo HAT focus. Their prevalences were $1.97 \%$ for $T$. congolense savannah type and $13.11 \%$ for T. brucei (s.l.). The highest infection rate of $T$. congolense savannah type (5.63\%) and T. brucei (s.l.) (36.36\%) were found at Ipono and Mabiogo, respectively, of the Campo HAT focus. The lowest infection rates of $T$. congolense savannah type $(1.42 \%)$ and $T$. brucei (s.l.) (7.41\%) were found at Akak and Campo beach, respectively.

Trypanosoma congolense forest type was identified in tsetse caught in the Campo and Fontem HAT foci. Its prevalence was $5.57 \%$ in tsetse of the Campo HAT focus and $26.04 \%$ in those of the Fontem HAT focus.

Thirty-five (14.11\%; 32/248) co-infections comprising 5 triple and 30 double infections were observed. The double infections included $18(7.26 \%)$ T. congolense forest type + T. vivax, $8(3.22 \%)$ T. brucei (s.l. $)+T$. vivax, 2 $(0.81 \%) T$. congolense forest type $+T$. brucei (s.l.), 1 $(0.4 \%) T$. congolense forest type $+T$. congolense savannah type, and $1(0.4 \%) T$. vivax $+T$. congolense savannah type. The five triple infections were composed of 3 (1.21\%) infections with $T$. congolense forest type $+T$. vivax + T. brucei (s.l.), $1(0.4 \%)$ with T. congolense forest type $+T$. congolense savannah type $+T$. vivax, and 1 $(0.4 \%)$ with $T$. congolense forest type $+T$. congolense savannah type $+T$. brucei (s.l.).

\section{Wolbachia and trypanosome co-infection}

From tsetse flies that were simultaneously analyzed for the presence of Wolbachia and different trypanosome species, 25.4\% (198/779) harbored Wolbachia and 31.8\% (248/779) were infected with at least one trypanosome species. Considering the fact that $T$. vivax is found exclusively in the mouthparts, single infections involving only this parasite were excluded from association studies between Wolbachia and trypanosomes. With these criteria, 125 flies with single infection of $T$. vivax were excluded. The 31 flies with double and triple infections involving $T$. vivax were considered for association studies. With the exclusion of 125 flies with single infections of $T$. vivax, only 123 tsetse flies with trypanosome infections were subjected to association studies. The Bipindi HAT focus was also excluded from these investigations because only infections due to $T$. vivax were found in tsetse caught in this focus. From 123 flies with trypanosome infections, 37 (30.08\%) hosted Wolbachia $(\mathrm{W}+\mathrm{T}+)$ while the remaining $86(69.92 \%)$ were devoid of Wolbachia (W-T+) (Table 4). Fifty-six tsetse flies hosting Wolbachia were infected by $T$. vivax. These 56 flies were also excluded and consequently only 144 tsetse flies $(\mathrm{W}+)$ hosting Wolbachia were considered for association studies. No trypanosomes were identified in $38.89 \%(56 / 144)(\mathrm{W}+\mathrm{T}-)$ of these 144 flies. A total of $340(59.65 \%)$ tsetse flies (W-T-) were devoid of infection with either trypanosome or Wolbachia (Table 4).

A generalized linear model (glm), used to test whether the presence of Wolbachia could have impact on the trypanosome infections, revealed no significant association between the two microorganisms in the Campo HAT focus $(r=0.009, P=0.993 ; 95 \% \mathrm{CI}:-0.77-0.71)$ and in the Fontem HAT focus $(r=1.403, P=0.161 ; 95 \%$ CI: -0.17-0.99) (Table 4).

\section{Discussion}

The entomological surveys revealed four tsetse subspecies, with G. p. palpalis being the predominant subspecies in the three HAT foci. These data confirm previous reports and highlight not only the great adaptability of $G$. p. palpalis, but also the fact that it is the main vector of African trypanosomes in southern Cameroon [17, 29-32]. The variation of tsetse subspecies according to HAT foci (only G. p. palpalis at Fontem, G. p. palpalis and G. p. pallicera at Bipindi and four species at Campo) indicates how the bio-ecological and bioclimatic conditions characterizing each HAT focus may have impacts on the tsetse fauna [30-32]. The unusual presence of G. tabaniformis suggests either an advance of human activity towards areas where it is usually confined or its incursion into anthropized areas where they can easily find vertebrate hosts for their blood meals.

The identification of Wolbachia in G. p. palpalis contrasts with results of Cheng et al. [15] and Doudoumis et al. [13] who did not identify Wolbachia in G. p. palpalis as in other tsetse of the fuscipes group. Our results are in line with those of Schneider et al. [33] who used sensitive PCR based-methods and identified Wolbachia in G. $f$. fuscipes. The sensitivity of molecular markers and the method used to detect Wolbachia are important factors that can affect Wolbachia infection rates. In our study where two markers (16S rDNA and WSP) were used, neither was more sensitive because no significant difference $(P=0.53)$ was observed between their overall performances. However, between HAT foci, significant differences were observed in the infection rates identified by these markers. For instance, WSP was two-fold more sensitive in the Campo focus $(20.19 \%$ of infections with WSP against $9.45 \%$ for $16 \mathrm{SDNA}$ ) while $16 S$ rDNA showed higher sensitivity $(25.37 \%$ against $6.93 \%$ for WSP) in the Fontem HAT focus. If one marker had been used, the overall Wolbachia prevalence would have been approximately $15.32 \%$ for WSP and $16.45 \%$ for $16 \mathrm{~S} \mathrm{rDNA}$. Each single marker underestimated the 
Table 4 Combined results of Wolbachia and trypanosome infections according to different HAT foci

\begin{tabular}{lllllllllll}
\hline Focus & No. of flies analyzed & W+T- & W+T+ & W-T+ & W-T- & W+ & T+ & $r$ & $P$-value of glm & $95 \%$ Cl \\
\hline Campo & 305 & 51 & 11 & 43 & 200 & 62 & 54 & 0.009 & 0.993 & $-0.768-0.705$ \\
Fontem & 265 & 56 & 26 & 43 & 140 & 82 & 69 & 1.403 & 0.161 & $-0.171-0.987$ \\
Total & 570 & 107 & 37 & 86 & 340 & 144 & 123 & & & \\
\hline
\end{tabular}

Abbreviations: $W+$ tsetse flies infected with Wolbachia sp., $T+$ tsetse flies infected with at least one trypanosome species, $W+T+$ tsetse flies co-infected with Wolbachia sp. and at least one trypanosome species, $W+T$ - tsetse flies infected with Wolbachia sp. but without trypanosome infection, $W$ - $T+$ tsetse flies without Wolbachia sp. but infected with at least one trypanosome species, $W$ - $T$ - tsetse flies infected with neither Wolbachia sp. nor trypanosome species, $C l$ confidence interval, $g / m$ generalized linear model, $r$ generalized linear model coefficient

Wolbachia prevalence since about $9 \%$ of infections would have not been detected by each of them. The use of two markers improved the detection of Wolbachia. Our results suggest the combination of two markers for accurate identification of Wolbachia and the need to develop new bio-makers for reliable detection of Wolbachia infections. The identification of Wolbachia in natural populations of G. $p$. palpalis has important implications for the development of new strategies for vector control. With its ability to induce cytoplasmic incompatibility and to be transmitted from mother to offspring, Wolbachia can be genetically modified in order to produce bio-molecules that can interfere with the establishment and/or development of trypanosomes in tsetse flies. This can affect the vectorial competence of tsetse and disease transmission could be blocked through the genetically modified Wolbachia strains that conferred resistance to tsetse fly.

The overall Wolbachia infection rate of $25.32 \%$ is lower than the $44.3 \%, 98 \%$ and $100 \%$ reported in G. $f$. fuscipes [14], G. austeni [15] and G. m. morsitans [13], respectively. These differences could be related to specific biological characteristics of each tsetse subspecies. Indeed, for identical stimulus, interactions between tsetse and its symbiotic microorganisms vary with biological response of each tsetse subspecies. Such variations affect interactions between tsetse and its symbionts and, consequently, the Wolbachia infection rates. Variations of analytical methods could also explain these differences. In the present study, whole tsetse was used, while in other studies investigations were performed on isolated tissues. It is also plausible that there is a low density of Wolbachia in G. p. palpalis as already reported in Rhagoletis cerasi [34] and Drosophila paulistorum [35]. This hypothesis is strengthened by results of Wamwiri et al. [12] where G. austeni populations from Kenya had a high density of Wolbachia compared to those of South Africa. This low density could explain results of Doudoumis et al. [13] reporting no Wolbachia in G. p. palpalis.

The differences in the Wolbachia infection rates according to HAT foci are in line with observations reported elsewhere $[12,13,15]$. These differences could be related to eco-climatic conditions characterizing each focus. Although the three HAT foci are all located in the forest region of southern Cameroon, each of them is characterized by specific environmental and bio-climatic conditions that have impacts on tsetse biology, its symbiotic microorganisms and finally on the interactions between tsetse and its symbionts. Between villages of the same HAT focus, the differences observed in the Wolbachia infection rates can be linked to specific microclimates encountered in each village. This hypothesis is in line with observations reporting that in habitats where environmental conditions fluctuate slightly, the interaction between tsetse and its symbiotic microorganism is stable as well as the transmission of symbionts from mother to offspring [15]. A better understanding of the vector competence of tsetse requires considering the variability of biotopes within and between tsetse infested regions.

The identification of different trypanosomes confirms previous results $[31,32,36]$ and indicates current transmission of these parasites. The co-existence of $T$. congolense forest and savannah types indicates that the geographical limit ( $T$. congolense savannah and forest in the savannah and forest zones, respectively) tends to change with time. Between HAT foci, the significant differences observed in the trypanosome infection rates could be explained by the fauna composition and the contact frequency between tsetse and mammals.

Regardless of the HAT focus considered here, an increase was observed when our trypanosome infection rates were compared with those previously generated in the same HAT foci $(35.1 \%$ against $32.4 \%$ at Campo in 2008; 20.09\% against $9.8 \%$ at Bipindi in 2010 and $37.73 \%$ against $6.3 \%$ at Fontem in 2006) [17, 31, 37]. These results could be explained by the fact that whole tsetse was investigated in our study while previous investigations were undertaken mostly on tsetse midguts. However, the number of flies with immature, mature infections and mouth part infections are unknown with our approach.

In addition to simple infections, our results showed that approximately $14.11 \%$ of tsetse of southern Cameroon carried mixed infections of different trypanosome species. These results are in agreement with those of previous studies reporting mixed infections in animals 
and different tsetse subspecies of Cameroon [36-38] and other African countries [39-41]. Since whole tsetse was analyzed, no information could be inferred from the part of tsetse that was co-infected by trypanosomes. It is difficult to know the proportion of mature co-infections of different trypanosome species. Remarkably, some trypanosomes of triple infections ( $T$. congolense forest type, $T$. congolense savannah type and T. vivax) can be found in their mature forms in tsetse mouthparts. This highlights a high probability that several trypanosome species might be transmitted by a tsetse fly during a single blood meal on a vertebrate host. In such context, further investigations are required to understand which parasite will establish in the host and how mixed infections will impact the trypanosome transmission dynamics and animal health. With a high number of double and triple infections, there is a need to understand the evolution of these infections and their potential impacts on the transmission dynamics of trypanosomes.

The high trypanosome infection rates indicate not only their high transmission in the forest regions of southern Cameroon, but also the need to implement and intensify control operations to achieve HAT elimination and reduce the incidence of AAT. The identification of trypanosomes in whole tsetse generated more data on trypanosome infections and highlighted AAT as threat for animal health in HAT foci of the southern Cameroon.

The $29.84 \%$ of tsetse with co-infections of Wolbachia and trypanosomes corroborates results of Alam et al. [14] and Aksoy et al. [42]. This relatively low co-infection rate can be related to the biological effects that this bacterium has on various parasites [14]. The absence of significant correlation between Wolbachia and trypanosome infections (Table 4) suggests that the presence of Wolbachia does not seem to be an obstacle for the establishment of trypanosomes. This result contrasts the negative correlation reported in G. f. fuscipes by Alam et al. [14] who subsequently suggested the prevention of trypanosome infections by the presence of Wolbachia. The tripartite association between tsetse, Wolbachia and trypanosomes seems to vary according to tsetse subspecies and tsetse populations. Obtaining an overview of the vector competence of tsetse requires also taking into consideration the teneral status of tsetse and its first blood meal on a non-infected host because these factors affect its ability to be infected and could mitigate the influence of symbiotic microorganisms.

With the differences observed in the sensitivity of markers and the presence of tsetse with Wolbachia infections and/or without trypanosomes, additional investigations on these bacteria are needed. Instead of focusing only on the presence/absence of Wolbachia, investigations aiming to characterize Wolbachia and to determine its density could generate additional data that may help to better understand Wolbachia infections as well as the contribution of this bacterium in the vector competence of tsetse flies. Moreover, as Wolbachia is maternally transmitted from mother to offspring, studies on population genetics of tsetse coupled with Wolbachia identification could enable to understand the differences in the susceptibility of different tsetse genotypes to Wolbachia infections.

\section{Conclusions}

The present study reveals significant differences in the infections rates of Wolbachia and trypanosomes in G. p. palpalis from HAT foci of southern Cameroon. The identification of Wolbachia with only one marker underestimates its infection rates and the combination of several markers enables achieving higher accuracy. Co-infections of Wolbachia and trypanosomes are not common and no association between these two microorganisms was revealed in G. p. palpalis. The tripartite association between tsetse fly, Wolbachia and trypanosomes seems to vary according to tsetse infested areas and a better understanding of this association may require additional studies aiming to evaluate the genetic polymorphism of Wolbachia as well as its density in tsetse flies.

\section{Abbreviations}

AAT: Animal African trypanosomiasis; HAT: Human African trypanosomiasis; rDNA: Ribosomal DNA; WSP: Wolbachia surface protein

\section{Acknowledgements \\ This work was supported by IRD through Jeune Equipe de Recherche Associée (JEAI EpiReTryp), UMR INTERTRYP and the University of Dschang. \\ Funding \\ JEAI EpiReTryp, UMR INTERTRYP and the Fond institutionnel d'appui à la recherche de I'Université de Dschang, Cameroon.}

Availability of data and materials

All data generated and/or analyzed during this study are included in this article.

Authors' contributions

SK was involved in sample collection, identification of symbionts and trypanosomes, and in drafting the manuscript. EO and TM participated in the sampling. FN revised the manuscript. GS participated in tsetse fly sampling, conception and design of the study, and drafting the manuscript. All authors read and approved the final manuscript.

\section{Ethics approval}

This study was carried out in strict accordance with the recommendations in the Guide for the Care and Use of Animals of the Department of Biochemistry, University of Dschang, Cameroon.

Consent for publication

Not applicable.

\section{Competing interests}

The authors declare that they have no competing interests.

\section{Publisher's Note}

Springer Nature remains neutral with regard to jurisdictional claims in published maps and institutional affiliations. 


\section{Author details}

'Molecular Parasitology and Entomology Unit (MPEU), Department of Biochemistry, Faculty of Science, University of Dschang, PO Box 67, Dschang, Cameroon. ${ }^{2}$ Laboratory of Parasitology and Ecology, Faculty of Science, University of Yaoundé I, Yaoundé, Cameroon.

Received: 17 August 2018 Accepted: 23 November 2018

Published online: 12 December 2018

\section{References}

1. World Health Organization. Control and surveillance of human African trypanosomiasis: report of WHO expert Committee. Geneva: World Health Organization; 2013

2. Fèvre EM, Picozzi K, Jannin J, Welburn SC, Maudlin I. Human African trypanosomiasis: epidemiology and control. Adv Parasitol. 2006;61:56.

3. Büscher P, Cecchi G, Jamonneau V, Priotto G. Human African trypanosomiasis. Lancet. 2017;390:2397-409.

4. Franco JR, Cecchi G, Priotto G, Paone M, Diarra A, Grout L, et al. Monitoring the elimination of human African trypanosomiasis: update to 2014. PLoS Negl Trop Dis. 2017;11:e0005585

5. Werren JH, Zhang W, Guo LR. Evolution and phylogeny of Wolbachia: reproductive parasites of arthropods. Proc Biol Sci. 1995;261:55-71.

6. Marcon HS, Coscrato VE, Selivon D, Perondini ALP, Marino CL. Variations in the sensitivity of different primers for detecting Wolbachia in Anastrepha (Diptera: Tephritidae). Braz J Microbiol. 2011;42:778-85.

7. Werren JH, Baldo L, Clark ME. Wolbachia: master manipulators of invertebrate biology. Nat Rev Microbiol. 2008;6:741-51.

8. Martinez J, Tolosana I, Ok S, Smith S, Snoeck K, Day JP, et al. Symbiont strain is the main determinant of variation in Wolbachia-mediated protection against viruses across Drosophila species. Mol Ecol. 2017;26:4072-84.

9. Yen JH, Barr AR. The etiological agent of cytoplasmic incompatibility in Culex pipiens. J Invert Pathol. 1973;22:242-50

10. Sinkins SP. Wolbachia and cytoplasmique incompatibility in mosquitoes. Insect Biochem Mol Biol. 2004;34:723-9.

11. Balmand S, Lohs C, Aksoy S, Heddi A. Tissue distribution and transmission routes for the tsetse fly endosymbionts. J Invert Pathol. 2013;112(Suppl. ):S116-22.

12. Wamwiri NF, Alam U, Thande CP, Aksoy E, Ngure MR, Aksoy S, et al. Wolbachia, Sodalis and trypanosome co-infections in natural populations of Glossina austeni and Glossina pallidipes. Parasit Vectors. 2013;6:232.

13. Doudoumis V, Tsiamis G, Wamwiri F, Brelsfoard C, Alam U, Aksoy E, et al. Detection and characterization of Wolbachia infections in laboratory and natural populations of different species of tsetse flies (genus Glossina). BMC Microbiol. 2012;12(Suppl. 1):S3.

14. Alam U, Hyseni C, Symula R, Brelsfoard C, Wu Y, Kruglov O, et al. Implications of microfauna-host interactions for trypanosome transmission dynamics in Glossina fuscipes fuscipes in Uganda. Appl Env Microbiol. 2012;78:4627.

15. Cheng Q, Ruel TD, Zhou W, Moloo SK, Majiwa P, O'Neill SL, et al. Tissue distribution and prevalence of Wolbachia infections in tsetse flies, Glossina spp. Med Vet Entomol. 2000;14:44-50.

16. Geiger A, Ravel S, Mateille T, Janelle J, Patrel D, Cuny G, et al. Vector competence of Glossina palpalis gambiensis for Trypanosoma brucei s.l. and genetic diversity of the symbiont Sodalis glossinidius. Mol Biol Evol. 2007:24:102-9.

17. Farikou O, Njiokou F, Mbida Mbida JA, Njitchouang GR, Djeunga HN, Asonganyi T, et al. Tripartite interactions between tsetse flies, Sodalis glossinidius and trypanosomes-an epidemiological approach in two historical human African trypanosomiasis foci in Cameroon. Infect Genet Evol. 2010;10:115-21

18. Grébaut P, Bodo JM, Assona A, Foumane NV, Njiokou F, Ollivier G, et al. Recherche des facteurs de risque de la trypanosomiase humaine Africaine dans le foyer de Bipindi au Cameroun. Med Trop. 2001;61:377-83.

19. Njiokou $F$, Laveissière $C$, Simo $G$, Nkinin $S$, Grébaut $P$, Cuny $G$, et al. Wild fauna as probable animal reservoir for Trypanosoma brucei gambiense in Cameroon. Infect Genet Evol. 2006:6:147-53.

20. Gouteux JP, Lancien JC. The pyramidal trap for collecting and controlling tsetse flies (Dipteria: Glossinidae). Comparative trials and description of new collecting techniques. Trop Med Parasitol. 1986;37:61-6.

21. Pollock JN. Training Manual for tsetse control personnel: Tsetse biology, systematics and distribution; techniques. Rome: Food and agriculture organization of the United Nations; 1982. http://www.fao.org/3/ap5178e.pdf.

22. Navajas M, Lagnel J, Gutierrez J, Bourset P. Species wide homogeneity of nuclear ribosomal ITS2 sequences in the spider mite Tetranychus urticae contrasts with extensive mitochondrial COI polymorphism. Heredity. 1998:80:742-52

23. Werren $\mathrm{JH}$, Windsor DM. Wolbachia infection frequencies in insects: evidence of a global equilibrium? Proc R Soc Lond B Biol Sci. 2000:267:1277-85.

24. Baldo L, Hotopp JCD, Jolley KA, Bordenstein SR, Biber SA, Choudhury RR, et al. Multilocus sequence typing system for the endosymbiont Wolbachia pipientis. Appl Environ Microbiol. 2006;72:7098-110.

25. Herder S, Simo G, Nkinin S, Njiokou F. Identification of trypanosomes in wild animals from southern Cameroon using the polymerase chain reaction (PCR). Parasite. 2002:9:345-9.

26. Masiga DK, Smyth AJ, Ayes P, Bromidge TJ, Gibson WC. Sensitive detection of trypanosomes in tsetse flies by DNA amplification. Int J Parasitol. 1992;22:909-18

27. Moser DR, Cook GA, Ochs DE, Bailey CP, McKane MR, Donelson JE. Detection of Trypanosoma congolense and Trypanosoma brucei subspecies by DNA amplification using the polymerase chain reaction. Parasitol. 1989;99:57-66.

28. R Development Core Team. R: A language and environment for statistical computing. Vienna: R Foundation for Statistical Computing; 2017. ISBN 3-900051-07-0, URL: https://www.freestatistics.org/cran/.

29. Simo G, Njiokou F, Mbida Mbida A, Njitchouang GR, Herder S, Asongany T, et al. Tsetse fly host preference from sleeping sickness foci in Cameroon: epidemiologic implication. Infect Genet Evol. 2008:8:34-9.

30. Melachio Tanekou TT, Simo G, Ravel S, De Meeûs T, Causse S, Solano P, et al. Population genetics of Glossina palpalis palpalis from central African sleeping sickness foci. Parasit Vectors. 2011;4:140.

31. Tchouomene-Labou J, Nana-Djeunga H, Simo G, Njitchouang GR, Cuny G, Asonganyi $T$, et al. Spatial and temporal variations relevant to tsetse control in the Bipindi focus of southern Cameroon. Parasit Vectors. 2013;6:193.

32. Grébaut $P$, Melachio $T$, Nyangmang $S$, Ebo'o Eyenga $V$, Njitchouang GR, Ofon $\mathrm{E}$, et al. Xenomonitoring of sleeping sickness transmission in Campo (Cameroon). Parasit Vectors. 2016:9:201

33. Schneider DI, Kathrin IG, Andrew GP, Adly MM, Abd-Alla Wolfgang JM Global Wolbachia prevalence, titer fluctuations and their potential of causing cytoplasmic incompatibilities in tsetse flies and hybrids of Glossina morsitans subgroup species. J Invertebr Pathol. 2013;112(Suppl.):S104-15.

34. Arthofer W, Riegler M, Schneider D, Krammer M, Miller WJ, Stauffer C. Hidden Wolbachia diversity in field populations of the European cherry fruit fly, Rhagoletis cerasi (Diptera, Tephritidae). Mol Ecol. 2009:18:3816-30.

35. Miller WJ, Ehrman L, Schneider D. Infectious speciation revisited: impact of symbiont-depletion on female fitness and mating behavior of Drosophila paulistorum. PLoS Pathog. 2010;6:e1001214.

36. Morlais L, Grebaut P, Bodo JM, Djoha S, Cuny G, Herder S. Detection and identification of trypanosomes by polymerase chain reaction in wild tsetse flies in Cameroon. Acta Trop. 1998:70:109-17.

37. Simo G, Fogue SP, Melachio TT, Njiokou F, Kuiate JR, Asonganyi T. Population genetics of forest type of Trypanosoma congolense circulating in Glossina palpalis palpalis of Fontem in the South-West region of Cameroon. Parasit Vectors. 2014;7:385

38. Nimpaye $H$, Njiokou F, Njine T, Njitchouang GR, Cuny G, Herder $\mathrm{S}$, et al. Trypanosoma vivax, $T$. congolense forest type and $T$. simiae: prevalence in domestic animals of sleeping sickness foci of Cameroon. Parasite. 2011;18:171-9.

39. Onyekwelu KC, Ejezie FE, Eze AA, Ikekpeazu JE, Ezeh RC, Edeh GC Prevalence of trypanosome infection in tsetse flies from Oji River and Emene axis of Enugu state, Nigeria: a preliminary report. Trop Parasitol. 2017;7:98-102.

40. Malele I, Graske L, Knight C, Ferris V, Njiru Z, Zamilton P, et al. The use of specific and genetic primers to identify trypanosomes infectious of wild tsetse flies in Tanzania by PCR. Infect Genet Evol. 2003;3:271-9.

41. Masiga DK, McNamara JJ, Laveissière C, Truc P, Gibson WC. A high prevalence of mixed trypanosome infections in tsetse flies in Sinfra, Côte d'Ivoire, detected by DNA amplification. Parasitol. 1996;112:75-80.

42. Aksoy S, Caccone A, Galvani AP, Okedi LM. Glossina fuscipes populations provide insights for human African trypanosomiasis transmission in Uganda. Trends Parasitol. 2013;29:394-406. 